\title{
Morphology-dependent resonances of optical microsphere resonators for the realization of passive wavelength-division multiplexing components
}

\author{
Şenol iş̧çi* \\ Temel Bilici ${ }^{\dagger}$ \\ Adnan Kurt \\ Ali Serpengüzel, MEMBER SPIE \\ Koç University \\ Microphotonics Research Laboratory and \\ Department of Physics \\ Rumeli Feneri Yolu, Sariyer \\ Istanbul 34450 Turkey \\ E-mail: aserpenguzel@ku.edu.tr
}

\begin{abstract}
Morphology-dependent resonances of microspheres can provide the necessary optical feedback for applications in spectroscopy, laser science, and optical communications. The elastic scattering of focused light from dielectric microspheres is understood by the localization principle and the generalized Lorenz-Mie theory. We excited the morphology-dependent resonances of glass microspheres by a tunable distributed-feedback laser and detected the elastically scattered signal. Efficient coupling to morphology-dependent resonances is achieved using an optical fiber half coupler. Resonance peaks in the elastic scattering spectra and associated dips in the transmission spectra are observed experimentally. Simulation results of elastic scattering spectra of glass microspheres in the C-band are presented. (C) 2004 Society of Photo-Optical Instrumentation Engineers. [DOI: 10.1117/1.1689332]
\end{abstract}

Subject terms: channel dropping filter; microsphere resonator; morphologydependent resonances; whispering gallery modes; wavelength division multiplexing.

Paper WDM-05 received Mar. 30, 2003; revised manuscript received Dec. 2, 2003; accepted for publication Dec. 6, 2003.

\section{Introduction}

Optical microsphere resonators have found various applications in quantum optics, laser science, spectroscopy, and optoelectronics. ${ }^{1}$ Microspheres possess high-quality-factor ( $Q$-factor) optical morphology-dependent resonances (MDRs) and have relatively small volumes. ${ }^{2}$ These tiny optical cavities, whose diameters may vary from a few to several hundred micrometers, have MDRs with reported $Q$-factors as large ${ }^{3}$ as $3 \times 10^{9}$. Low-threshold lasing in Nddoped silica, ${ }^{4}$ polymer, ${ }^{5}$ and Raman active media ${ }^{6}$ have been demonstrated using microspheres. MDRs of dielectric microspheres provide the necessary optical feedback for applications in optical communications, such as optical couplers, optical amplifiers, and optical filters. Strain tunable microsphere oscillators, ${ }^{7}$ add-drop filters, ${ }^{8}$ and thermooptical switching in $\mu$-spheres ${ }^{9}$ have also been realized for filtering, multiplexing, and switching applications.

Microspheres are uniquely applicable in compact optoelectronic devices for wavelength-division multiplexing (WDM) applications. In optical communication, WDM offers a scalable solution for using existing fiber optic networks to gain increased bandwidth. More channels with reduced channel spacing are possible with dense WDM (DWDM) technology. In these lightwave circuits, dielectric

\footnotetext{
*Currently at the Scientific and Technical Research Council of Turkey (TUBITAK), National Institute of Electronics and Cryptology (UEKAE), P.K. 74, Gebze, Kocaeli 41470 Turkey.

Currently at Boston University, Department of Electrical and Computer

Engineering, 8 St. Mary's Street, Boston, Massachusetts, 02215 USA.
}

microspheres can be used to develop WDM and DWDM components, which support narrow channel spacing, high channel counts, and the addition and the dropping of a large number of wavelengths.

In this paper, MDRs are characterized experimentally by evanescently coupling light along an optical fiber half coupler (OFHC) into glass microspheres. We also analyzed the MDR spectra of solid microspheres in the C-band for possible WDM and DWDM applications.

\section{MDRs}

The MDRs of the $\mu$-sphere can be explained as follows: as the light propagates around the inside surface of the $\mu$-sphere, it undergoes an almost total internal reflection ${ }^{10}$ (TIR). The rays of the MDR have the property that all subsequent bounces have the same angle of incidence. After circumnavigating the $\mu$-sphere, the light wave returns to the starting point in phase to interfere constructively with itself. This constructive interference can occur only at certain discrete MDR wavelengths. ${ }^{11}$ Hence, the light is confined to a band within the great circle of the $\mu$-sphere. Each MDR is characterized by a mode number $(n)$ and a mode order $(l)$. Physically, $n$ indicates the number of nodes in the internal intensity distribution as the polar angle is varied from 0 to $180 \mathrm{deg}$. The mode order $l$ indicates the number of nodes in the internal intensity distribution in the radial direction. ${ }^{12}$ For each set of mode numbers,${ }^{13}$ there is a transverse electric (TE) and transverse magnetic (TM) MDR. For a given $\mu$-sphere, the MDR occurs at a specific value of the size parameter, $x_{n, l}$, which is given by 


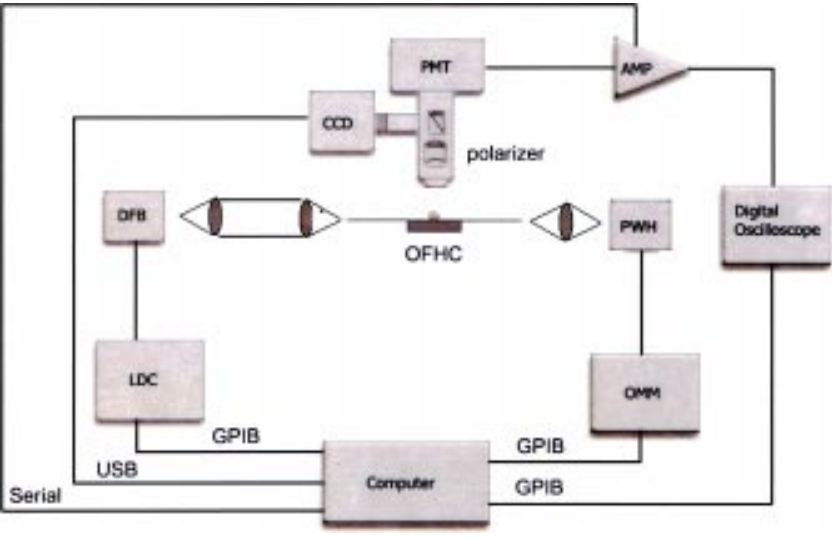

Fig. 1 Schematic of the experimental setup.

$2 \pi a / \lambda_{n, l}$, where $\lambda_{n, l}$ is the light wavelength in vacuum, and $a$ is the radius of the $\mu$-sphere. These MDRs have been verified experimentally at optical wavelengths with micrometer-sized spheres.

Many applications of the microspheres require coupling of light into MDRs from external light sources. The greatest impediment to the use of $\mu$-sphere resonators in practical devices has been the difficulty of efficiently light coupling into and out of the $\mu$-spheres. One way to excite MDRs is to use evanescent coupling method. To couple light into or out of the $\mu$-sphere, it is necessary to overlap the evanescent field of the MDR with the evanescent field of the TIR. Such coupling has been implemented, by the use of thin tapered fibers, OFHCs, angle-polished fibers, high-index prisms, and planar waveguide couplers. ${ }^{14}$ Fiber half couplers are also used to produce polarization splitters, pump/signal combiners, and loss compensators for optical fiber communications. If a $\mu$-sphere is placed on the exposed surface near the evanescent field of the fiber optic core, then there is an efficient energy exchange between the waveguide mode of the fiber and the MDR of the $\mu$-sphere. ${ }^{15}$

\section{Experimental Setup}

A schematic of the experimental setup is shown in Fig. 1. Light is coupled into the microsphere by using an OFHC. The OFHC is fabricated from a 810 -nm single-mode fiber with a core radius of $1.9 \mu \mathrm{m}$ and a refractive index of 1.462 , and a cladding radius of $62.5 \mu \mathrm{m}$ with refractive index of 1.457, which is laid on a glass substrate with a low curvature. ${ }^{16}$ The cladding of the fiber below the $\mu$-sphere is shaved down to $0.7 \mu \mathrm{m}$ to approach the core of the fiber. This excitation geometry effectively becomes the optical equivalent of a Gaussian beam with an infinite skirt length passing near the $\mu$-sphere. The transmission loss from the OFHC corresponds to $21 \mathrm{~dB}(0.8 \%)$. BK7 glass microspheres are used in the experiment, because glass exhibits low loss at optical communication wavelengths. The glass microsphere has a radius of $a=500 \mu \mathrm{m}$ and refractive index of 1.50. Excitation of MDRs of the microsphere is achieved by using a tunable distributed-feedback (DFB) semiconductor laser, whose center wavelength is $810 \mathrm{~nm}$. Wavelength tuning is achieved by tuning the temperature of the DFB laser with a laser diode controller (LDC). Both the
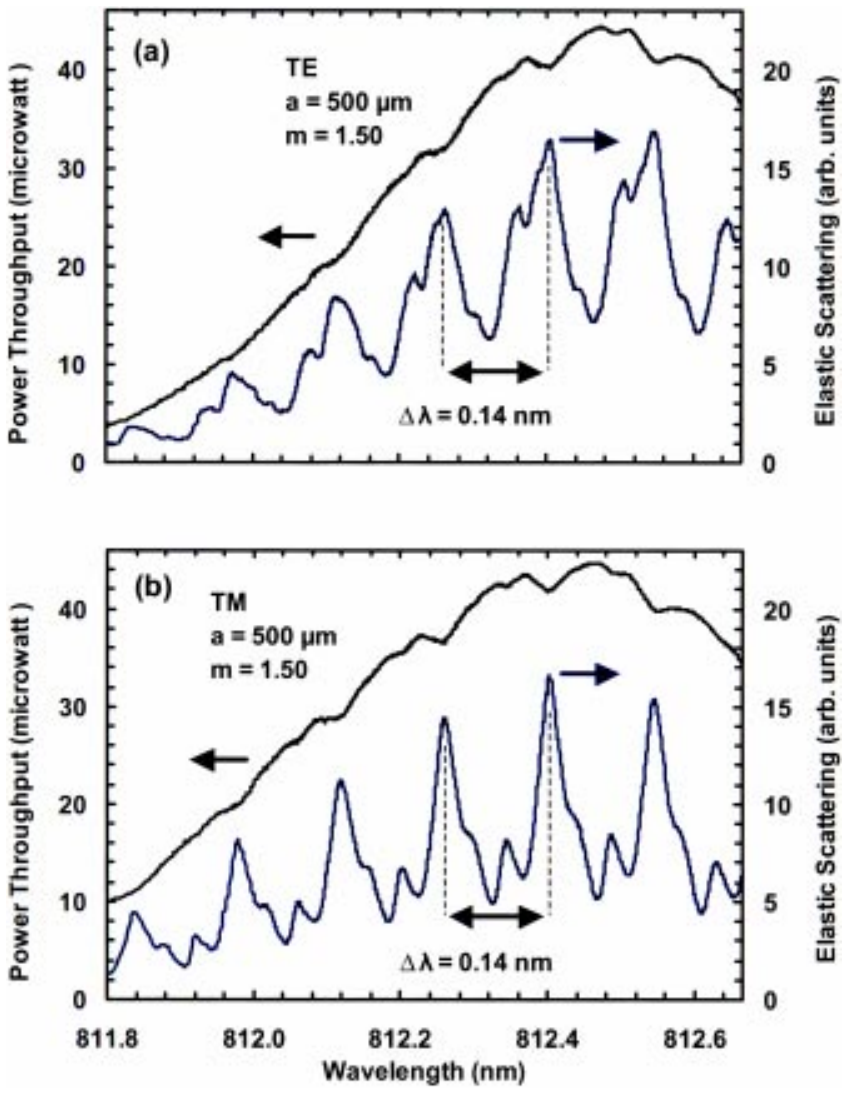

Fig. 2 Temperature tuning spectra of the power transmission (upper curve) and the elastic scattering (lower curve) when the polarizer is at (a) 90 and (b) 0 deg, respectively.

transmitted light through the optical fiber, and the scattered light from the $\mu$-sphere at a right angle $(90 \mathrm{deg})$ are detected. A polarizer is inserted between the microscope and photomultiplier tube (PMT) to separate the TE and the TM MDRs. The PMT signal is sent to a digital oscilloscope for signal monitoring and data acquisition. The optical power and the wavelength of the transmitted light are measured by an optical multimeter (OMM) with a silicon power/ wavehead (PWH). Device control and data acquisition are performed with standard IEEE-488 general purpose interface bus (GPIB) interface.

\section{Experimental Results}

Figure 2 shows the elastic scattering and the power transmission spectra obtained by temperature tuning of the DFB laser between 19 and $32^{\circ} \mathrm{C}$ at a constant current of 31.2 $\mathrm{mA}$. This temperature ranges corresponds to a wavelength range of 811.9 to $812.7 \mathrm{~nm}$. The spectral resolution of the acquisition system is determined to be $0.04 \mathrm{~nm}$. Figure 2(a) shows the elastic scattering spectrum of the TE MDRs, obtained through a polarizer with its polarization axis at 90 deg to the incoming beam. Figure 2(b) is obtained when the polarizer is placed at 0 deg to the incoming beam, corresponding to TM MDRs. We can clearly observe MDR peaks in the elastic scattering, and the associated dips in the power transmission spectra. In the spectra of Fig. 2, the linewidth (FWHM) of the MDRs of higher intensity is approximately $13 \mathrm{GHz}$ (i.e., $0.03 \mathrm{~nm}$ ), and the $\Delta \lambda$ of the 
higher intensity MDRs is measured to be $63 \mathrm{GHz}(0.14$ $\mathrm{nm}$ ), which is consistent with the calculation based on the Mie scattering theory given by

$\Delta \lambda=\left[\lambda^{2} \arctan \left(m^{2}-1\right)^{1 / 2}\right] /\left[2 \pi a\left(m^{2}-1\right)^{1 / 2}\right]$,

where $\Delta \lambda$ is defined as the separation between the adjacent peak wavelengths of the same mode order ( $l$ ) MDR's with subsequent mode numbers ${ }^{17}(n)$. Note that the fractional depth of the dips in the transmission spectra is not the same for all MDRs. This is due to different $Q$-factors and coupling efficiencies of the MDRs. However, the relative depth of the transmission dips is maintained at each $\Delta \lambda$. The broad Gaussian line shape of the power transmission spectra results from the coupling geometry of the DFB laser to the optical fiber. As the temperature of the DFB laser changes, the input laser spot moves with respect to the fiber core, when entering the OFHC.

\section{Coupling Efficiency}

Power coupled into the MDRs can be estimated from the power transmission spectra. For example, in the TM spectrum of Fig. 2(b), the MDR at $812.4 \mathrm{~nm}$ has a transmitted power of $40 \mu \mathrm{W}$. When the $\mu$-sphere is not present, the transmitted power is estimated to be $43 \mu \mathrm{W}$. Therefore, power coupled $\left(P_{i}\right)$ into the MDR is approximately $3 \mu \mathrm{W}$, which means a $6.9 \%$ (i.e., $0.31 \mathrm{~dB}$ ) coupling loss. The scattered intensity $I_{i}$ can be expressed ${ }^{18}$ as $I_{i}=P_{i} / \sigma$, where $\sigma$ is the scattering cross section of the MDR. For a Gaussian beam, the scattering cross section is defined to be $\sigma$ $=\omega_{0} \lambda / \sqrt{2}$, where $\omega_{0}$ is beam waist's half width. ${ }^{19}$ For our experimental parameters of $\omega_{0}=2 \mu \mathrm{m}$ and $\lambda=812.4 \mathrm{~nm}$, $I_{i}$ is calculated to be $0.75 \mathrm{~kW} / \mathrm{cm}^{2}$. We can also calculate the coupling efficiency of a Gaussian beam $\langle\sigma\rangle$ into MDRs using the localization principle, where $\langle\sigma\rangle=\sqrt{2} / \pi^{2} \omega_{0}$ is estimated to be 0.053 (i.e., 5.3\%), which compares favorably with the observed $6.9 \%$ coupling loss.

The measured quality $(Q)$ factor of the MDRs in the scattering spectrum is approximately of $10^{4}$. The high $Q$ factor MDRs are superimposed on a background of low- $Q$ factor MDRs. In practice, absorption, scattering on defects, scattering on impurities, and residual surface roughness ${ }^{18}$ are responsible for the measured losses corresponding to $Q$-factors due to the presence of small shape distortions, and surface irregularities of the particle. The observed $Q$-values should be compared with the values determined by $Q^{-1}=Q_{\mathrm{abs}}^{-1}+Q_{s}^{-1}+Q_{r}^{-1}$, where $Q_{r}^{-1}$ corresponds to the intrinsic diffraction leakage, $Q_{s}^{-1}$ is the losses due to light scattering by surface roughness and Rayleigh scattering inside the sphere, and $Q_{\text {abs }}^{-1}$ is the optical absorption loss. The presence of surface roughness and the molecular roughness would give higher-Q MDRs (Ref. 20), when the beam is aimed slightly inside the sphere's rim. ${ }^{21}$

\section{Theoretical Results}

For telecom applications of high channel counts, linewidths of the order of several gigahertz and channel spacing of several terahertz are necessary. Microspheres and MDRs can be tailored to enable required channel spacing and linewidths. Factors influencing the MDRs are mainly size and the refractive index of microspheres. To implement these
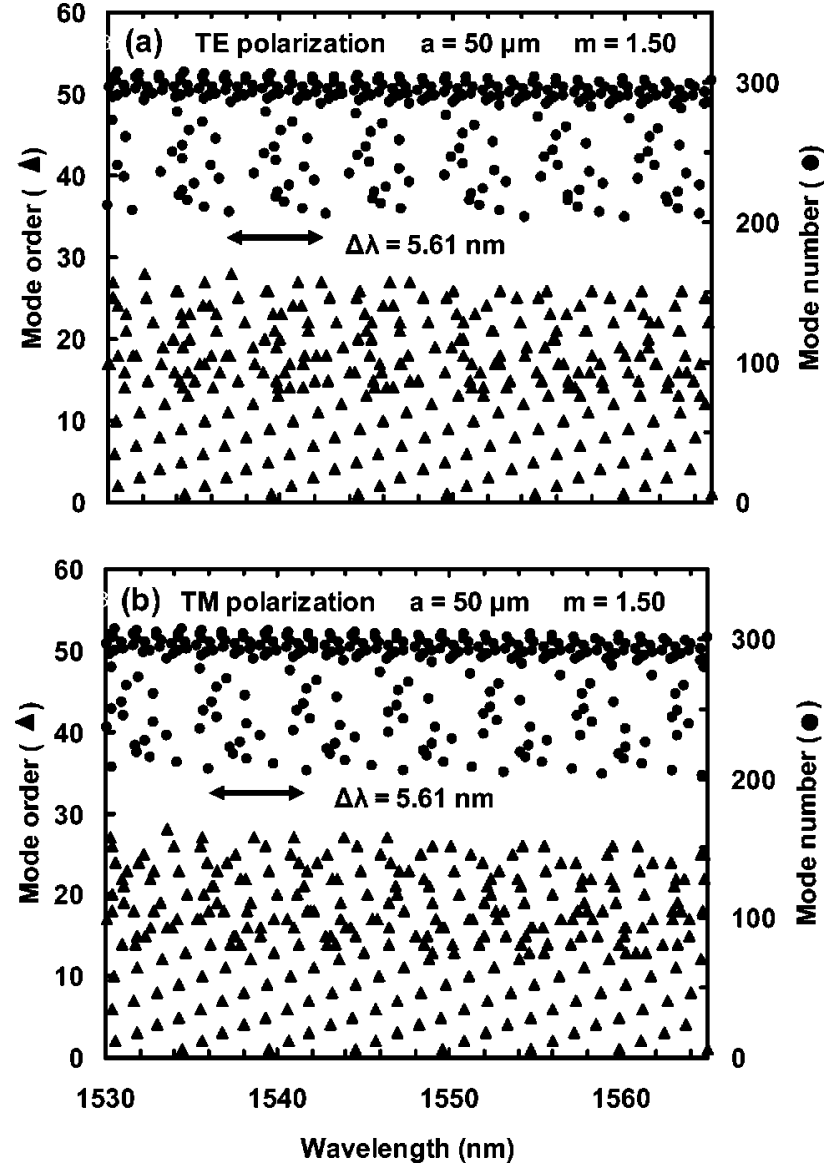

Fig. 3 Calculated (a) TE and (b) TM mode numbers ( $n$ ) and mode orders $(I)$ of the BK7 glass microsphere $(a=50 \mu \mathrm{m}, n=1.50)$ between 1530 and $1565 \mathrm{~nm}$.

criteria, we have also theoretically analyzed the MDR spectra of small solid microspheres in the C-band. Generalized Lorenz-Mie theory (GLMT), which is a direct extension of the plane-wave Lorenz-Mie theory, describes electromagnetic elastic scattering of an arbitrary light beam by a spherical particle. ${ }^{22}$ For the calculations of both TE and TM elastic scattering spectra, a Gaussian beam (with an infinite skirt length and beam waist with a half-width of $2 \mu \mathrm{m}$ ) propagating just at the edge of the microsphere is used ${ }^{23}$ at a scattering angle of $90 \mathrm{deg}$.

In Figs. 3(a) and 3(b), the mode and order numbers are calculated for both TM and TE MDRs at a wavelength range between 1530 and $1565 \mathrm{~nm}$, corresponding to C-band for a BK7 glass microsphere with the radius of $a=50 \mu \mathrm{m}$ and a refractive index of $m=1.50$, respectively. TE and TM elastic scattering spectra for glass microspheres with a radius of $50 \mu \mathrm{m}$ are calculated in Figs. 4(a) and 4(b). TE and TM elastic scattering spectra show observable MDRs. We also identified the mode numbers and mode orders of the MDRs. Most of the MDRs are not observable in the spectrum due to their high $Q$-factors. The $\Delta \lambda$ of subsequent mode orders is calculated to be $5.61 \mathrm{~nm}$. The $Q$-factors of the broadest MDRs are approximately $10^{4}$.

\section{Conclusions}

We demonstrated the excitation of MDRs of glass microspheres using an OFHC and a temperature-tunable DFB 

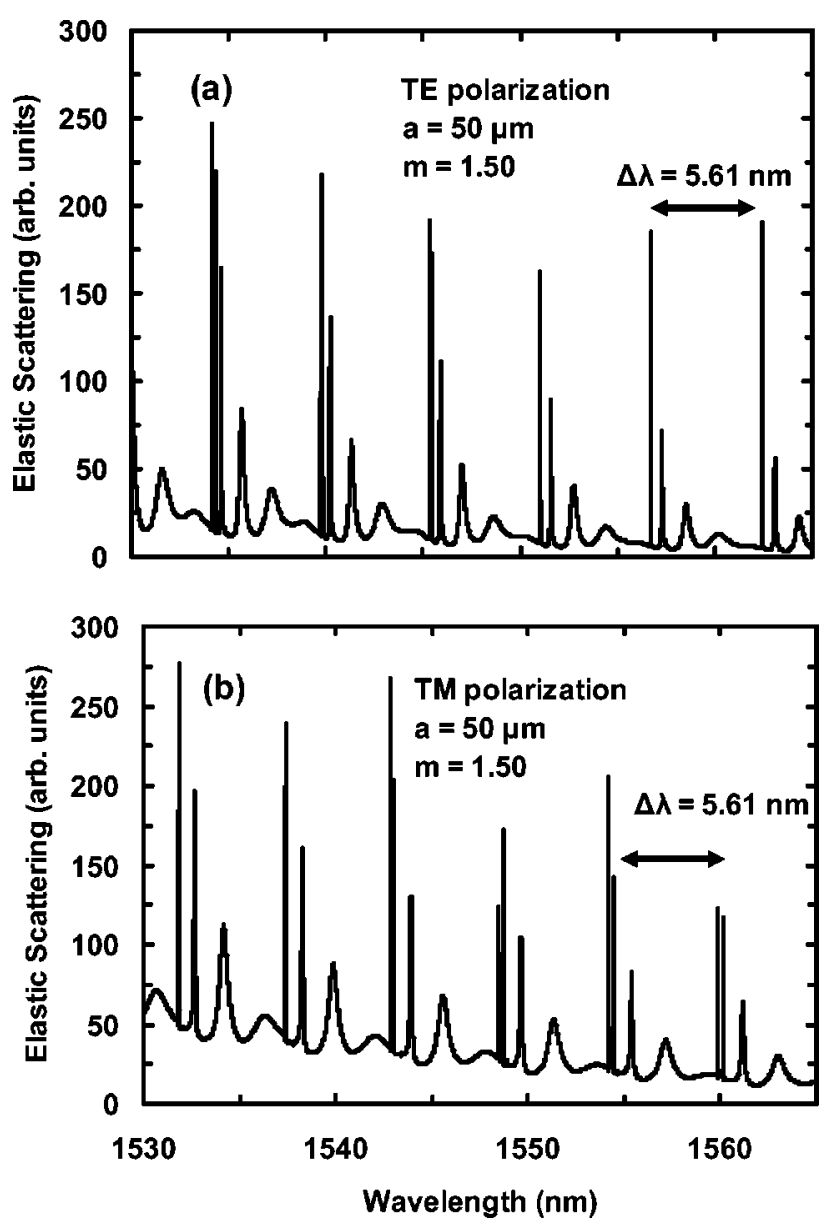

Fig. 4 Calculated elastic scattering spectra of the BK7 glass microsphere $(a=50 \mu \mathrm{m}, n=1.50)$ for (a) TE and (b) TM polarization.

laser. MDR peaks in the elastic scattering spectra and associated dips in the transmission spectra were observed experimentally. With the proper system design, it would be possible to totally drop the selected MDR powers from the transmission spectrum. The estimation of coupling efficiencies compares favorably with the experimentally measured values. Simulation results of elastic scattering spectra of glass microspheres in the $\mathrm{C}$-band for data transmission were presented. In conclusion, MDRs of microspheres can be exploited to develop passive multiplexing/ demultiplexing modules, optical add/drop multiplexers for WDM communications.

\section{Acknowledgments}

We would like to acknowledge the partial support of this research by the Scientific and Technical Research Council of Turkey (TUBITAK) Grant No: TBAG-1952 and the European Office of Aerospace Research and Development (EOARD) Grants No: F61775-01-WE062 and FA8655-02M-4086.

\section{References}

1. L. Collot, V. Lefèvre-Seguin, M. Brune, J. M. Raimond, and S. Haroche, "Very high-Q whispering-gallery mode resonances observed on fused silica microspheres," Europhys. Lett. 23, 327-334 (1993).

2. A. Serpengüzel, S. Arnold, and G. Griffel, "Excitation of resonances of microspheres on an optical fiber," Opt. Lett. 20(7), 654-656 (1995).

3. V. Lefèvre-Seguin and S. Haroche, "Towards cavity-QED experiments with silica microspheres," Mater. Sci. Eng. 48(1-2), 53-58 (1997).

4. V. Lefèvre-Seguin, "Whispering-gallery mode lasers with doped silica microspheres," Opt. Mater. (Amsterdam, Neth.) 11(2-3), 153165 (1999).

5. M. Kuwata-Gonokami and K. Takeda, "Polymer whispering gallery mode lasers," Opt. Mater. (Amsterdam, Neth.) 9(1-4), 12-17 (1998)

6. S. M. Spillane, T. J. Kippenberg, and K. J. Vahala, "Ultralowthreshold Raman laser using a spherical dielectric microcavity," $\mathrm{Na}$ ture (London) 415(6872), 621-623 (2002).

7. V. S. Ilchenko, P. S. Volikov, V. L. Velichansky, F. Treussart, V. Lefèvre-Seguin, J.-M. Raimond, and S. Haroche, "Strain-tunable high-Q optical microsphere resonator," Opt. Commun. 145(1-6), 86-90 (1998).

8. M. Cai, G. Hunziker, and K. J. Vahala, "Fiber-optic add-drop device based on a silica microsphere-whispering gallery mode system," IEEE Photonics Technol. Lett. 6(6), 686-687 (1999).

9. H. C. Tapalian, J. P. Laine, and P. A. Lane, "Thermooptical switches using coated microsphere resonators," IEEE Photonics Technol. Lett. 14(8), 1118-1120 (2002).

10. S. Arnold, "Microspheres, photonic atoms and the physics of nothing," Am. Sci. 89, 414-421 (2001).

11. B. R. Johnson, "Morphology-dependent resonances of a dielectric sphere on a conducting plane," J. Opt. Soc. Am. A 11(7), 2055-2064 (1994).

12. P. W. Barber and S. C. Hill, Eds., Light Scattering by Particles: Computational Methods, World Scientific, Singapore (1990).

13. R. K. Chang and A. J. Campillo, Eds., Optical Processes in Microcavities, World Scientific, Singapore (1996); P. W. Barber and R. K. Chang, Eds., Optical Effects Associated with Small Particles, World Scientific, Singapore (1988)

14. J.-P. Laine, B. E. Little, and H. A. Haus, "Etch-eroded fiber coupler for whispering-gallery mode excitation in high-Q silica microspheres," IEEE Photonics Technol. Lett. 11(7), 1429-1430 (1999).

15. A. Serpengüzel, S. Arnold, and G. Griffel, "Photonic atoms: enhanced light coupling," in Microcavities and Photonic Bandgaps: Physics and Applications, J. Rarity and C. Weisbuch, Eds., Kluwer Academic, Netherlands (1995).

16. G. Griffel, S. Arnold, D. Taskent, A. Serpengüzel, J. Connolly, and N Morris, "Morphology-dependent resonances of a microsphere-optical fiber system," Opt. Lett. 21(10), 695-697 (1996).

17. R. Jia, D. Jiang, P. Tan, B. Sun, J. Zhang, and Y. Lin, "Photoluminescence study of $\mathrm{CdSe}_{x} \mathrm{~S}_{1-x}$ quantum dots in a glass spherical microcavity," Chin. Phys. Lett. 18(10), 1350-1352 (2001).

18. W. V. Klitzing, R. Long, V. S. Ilchenko, J. Hare, and V. LefèvreSeguin, "Tunable whispering gallery modes for spectroscopy and CQED experiments," New J. Phys. 3(14), 1-14 (2001)

19. A. Serpengüzel, S. Arnold, G. Griffel, and J. A. Lock, "Enhanced coupling to microsphere resonances with optical fibers," J. Opt. Soc Am. B 14(4), 790-795 (1997)

20. J. P. Barton, "Effects of surface perturbations on the quality and the focused-beam excitation of microsphere resonance," J. Opt. Soc. Am A 16(8), 1974-1980 (1999).

21. H.-B. Lin, A. J. Campillo, and J. P. Barton, "Excitation localization principle for spherical microcavities," Opt. Lett. 23(24), 1921-1923 (1998).

22. J. A. Lock and G. Gouesbet, "Rigorous justification of the localized approximation to the beam shape coefficients in generalized LorenzMie theory. I. On-axis beams," J. Opt. Soc. Am. A 11(9), 2503-2515 (1994).

23. G. Gouesbet and J. A. Lock, "Rigorous justification of the localized approximation to the beam shape coefficients in generalized LorenzMie theory. II. Off-axis beams," J. Opt. Soc. Am. A 11(9), 2516-2525 (1994).

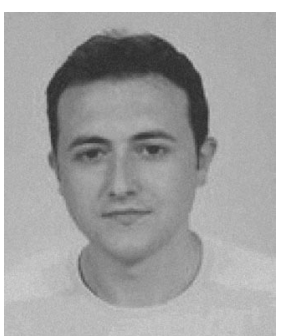

Şenol Işçi received his BS degree in physics and his MSc degree in biomedical engineering from Bogaziçi University, Istanbul, Turkey, in 2000 and 2003, respectively. During his master's study, he participated in research at Microphotonics Research Laboratory, Koç University, Istanbul, Turkey. He was also a research assistant with the Medical Imaging Laboratory of Biomedical Engineering Institute, Boğaziçi University, from 2001 to 2003. He is now doing his $\mathrm{PhD}$ work in biomedical engineering and is a researcher with National Institute of Electronics and Cryptology, Kocaeli, Turkey. His research interests include optical diagnostics and sensory devices, microcavity spectroscopy, and medical image processing. 
$\mathrm{He}$ is a member of the IEEE, the OSA, SPIE, and the International Society for Magnetic Resonance in Medicine (ISMRM).

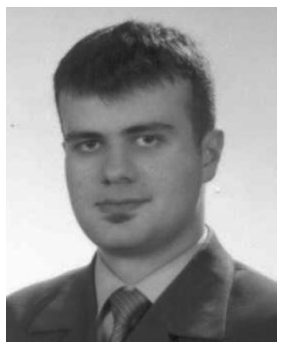

Temel Bilici received his BSc and MSc degrees from the Electrical and Electronics Engineering Department, Boğaziçi University, in 2000 and 2003, respectively, and is currently pursuing his $\mathrm{PhD}$ degree with the Electrical and Electronics Engineering Department, Boston University. He was also a research and teaching assistant with the Physics Department, of Koc University, in 2000 and was with the Koc University Microphotonics Research Laboratory. At Koç University, he mainly focused on microcavity photoluminescence experiments and elastic light scattering in dielectric microspheres for frequency control and filtering applications. $\mathrm{He}$ is the founder and the first president of the Koç University OSA Student Chapter. He is also a student member of the IEEE-CS and IEEE-LEOS.

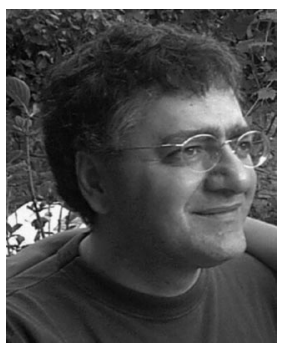

Adnan Kurt received his BSc degree in electrical engineering and his MSc degree in physics from Boğaziçi University in 1984 and 1987, respectively. He was a research assistant with Boğaziçi University, in the Physics Department and in the Psychology Department, performing research on laser speckle imaging, pulsed-gas laser design, and neurocomputing and animal learning from 1985 to 1990 . Later he joined the research group at the Center for Electroneurophysiology, Istanbul University Medical School, and engaged in human brain and neurophysiology research from 1990 to 1995 He was with Mitra A.S., Istanbul, a company operating in the area of printing, imaging, and electrophotography. Afterward, in 1999 he became a research engineer with the Koç University, Physics Department. There he is taking part in research programs on solid state laser design, optical and electronic instrumentation, and laser spectroscopy.

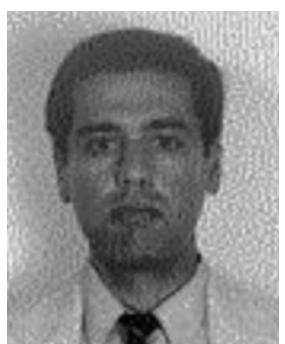

Ali Serpengüzel received his BSc degrees in electrical engineering and physics from Boğaziçi University, and his MSc, MPhil, and $\mathrm{PhD}$ degrees in applied physics from Yale University. He was later a research associate with Yale University continuing his research on nonlinear optics in microcavities. He was a consultant to Texaco Research Center. He joined Polytechnic University, Microparticle Photophysics Laboratory, as a postdoctoral research as sociate. He was an assistant professor with Bilkent University, Physics Department, Ankara, where he concentrated his research on semiconductor microcavities and photonic bandgap materials. He is a consultant to the Scientific and Technical Research Council of Turkey. $\mathrm{He}$ is currently an associate professor with Koç University, Physics Department, where he is continuing his research on microwave, optoelectronic, and microphotonic devices. He is a senior member of the IEEE and a member of the SPIE, OSA, APS, and Sigma-Xi. 